\title{
Modifications of gastric inhibitory polypeptide (GIP) secretion in man by a high-fat diet
}

\author{
BY L. M. MORGAN ${ }^{1}$, S. M. HAMPTON 1 , J. A. TREDGER ${ }^{1}$, R. CRAMB \\ AND V. MARKS ${ }^{1}$ \\ ${ }^{1}$ Department of Biochemistry, University of Surrey, Guildford, Surrey, GU2 $5 X H$ \\ ${ }^{2}$ St Luke's Hospital, Guildford, Surrey
}

(Received 9 July 1987 - Accepted 20 January 1988)

\begin{abstract}
1. Five healthy volunteers (usual fat intake 103 (SE 9) $\mathrm{g} / \mathrm{d}$ and energy intake 9855 (SE 937) kJ/d were given on two separate occasions (a) $100 \mathrm{~g}$ oral glucose and (b) sufficient intravenous (IV) glucose to obtain similar arterialized plasma glucose levels to those after oral glucose.

2. Subjects increased their fat intake by 68 (SE 9.6) \% for $28 \mathrm{~d}$ by supplementing their diet with $146 \mathrm{ml}$ double cream/d (fat intake on high-fat diet (HFD) 170 (SE 8) g/d; energy intake 12347 (SE 770)).

3. The $100 \mathrm{~g}$ oral glucose load was repeated and IV glucose again given in quantities sufficient to obtain similar arterialized blood glucose levels. Immunoreactive plasma insulin, C-peptide and gastric inhibitory polypeptide (GIP) were measured.

4. Plasma GIP levels were higher following oral glucose after the HFD (area under plasma GIP curve 0 $180 \mathrm{~min} 1660$ (SE 592) v. 2642 (SE 750) ng/l.h for control and HFD respectively; $P<0.05$ ). Both insulin and C-peptide levels were significantly higher after oral than after IV glucose $(P<0 \cdot 01)$ but neither were affected by the HFD. Glucose levels were lower following the HFD after both oral and IV glucose (area under plasma glucose curve 0-180 min, following oral glucose 6.7 (SE 0.3) mmol/1.h for control and 4.2 (SE 0.6) mmol/1.h for HFD; $P<0.01)$.

5. Glucose-stimulated GIP secretion was thus enhanced by the HFD. Insulin secretion in response to oral glucose was unchanged, in spite of an improvement in glucose tolerance.

6. The improvement in glucose tolerance post-HFD could possibly be due to a GIP-mediated inhibition of hepatic glycogenolysis, or a decreased rate of glucose uptake from the small intestine.
\end{abstract}

It is well established that intrajejunally- (McIntyre et al. 1964) or orally- (Elrick et al. 1964) administered glucose leads to significantly higher circulating insulin levels than does intravenous (IV) glucose producing comparable degrees of hyperglycaemia. Whilst higher levels of insulin after oral glucose could be due in part to decreased hepatic extraction of insulin (Gibby \& Hales, 1983), we have recently shown that they are largely due to an increased secretion of insulin (Hampton et al. 1986). The intestinal hormone gastric inhibitory polypeptide (GIP) is a potent stimulator of glucose-induced insulin secretion (Brown et al. 1975) and is currently thought to be one of the main components of the entero-insular axis.

Hyperinsulinaemia is a condition which has been implicated in the pathology of various disease states in which dietary factors also play a part, for example, coronary heart disease, maturity-onset diabetes and obesity (Stout, 1982). Whilst the causes of hyperinsulinaemia are still obscure, an overactive entero-insular axis could be implicated, at least in part, in the development of hyperinsulinaemic states. Exaggerated GIP responses have been observed after a mixed meal in obese subjects (Ebert et al. 1976) but these are reversed by $5 \mathrm{~d}$ of energy restriction (Willms et al. 1978). Exaggerated GIP responses to sucrose have been found in normal subjects after consuming a diet high in sucrose (O'Dorisio \& Cataland, 1981). We have previously shown in rats that a high-fat diet (HFD) causes both increased GIP and insulin secretion and some degree of insulin resistance (Hampton et al. 1983). The findings provide evidence that diet can be of considerable importance in changes in the activity of the entero-insular axis and the development of insulin resistance. 
However, a normal laboratory rodent diet, unlike the typical diet consumed by people in the Western world, contains very little fat and caution must be exercised when extrapolating the relevance of animal experiments to man.

We have therefore investigated the effect of an HFD in man on GIP secretion, and the contribution of the entero-insular axis to insulin secretion, by monitoring GIP and insulin concentrations following the induction of hyperglycaemia by oral and IV glucose both before and after the period of dietary modification. Doses of IV glucose were adjusted to produce similar degrees of hyperglycaemia in arterialized blood, as occurred after oral glucose.

\section{MATERIALS AND METHODS}

\section{Protocol}

Five healthy volunteers (four men and one woman), aged 26 (SE 1.5 ) years, all within $10 \%$ ideal body-weight and habitually consuming less than $125 \mathrm{~g}$ fat/d, participated in the study. For $7 \mathrm{~d}$ before the study, subjects kept a food diary in which they recorded all food and drink consumed in metric or household measures, or both, which were converted to weights before analysis. Subjects were encouraged during this time to adhere to their normal diet. For the next $35 \mathrm{~d}$, at intervals of $3 \mathrm{~d}$, subjects were given sufficient pots of double cream to incorporate $146 \mathrm{ml}$ double cream ( $68 \mathrm{~g}$ fat) into their diet daily. At each $3 \mathrm{~d}$ interview they brought back the empty pots (as a crude measure of compliance) and their completed food diaries.

If any subject felt full they were advised how to reduce their energy intake by reducing their consumption of foods low in fat thus maintaining a total fat intake $>150 \mathrm{~g} / \mathrm{d}$.

The food diaries were analysed using the University of Surrey superdiet computer program based on food composition tables (Paul \& Southgate, 1978).

Subjects underwent investigation on four separate occasions, two before and two during the $35 \mathrm{~d}$ dietary modification. After an overnight fast, two intravenous cannulas were inserted; one into an antecubital vein (for the collection of venous blood), the other into a dorsal vein of the ipsilateral hand heated to approximately $55^{\circ}$ by means of an electric heating blanket. This vein was used for the collection of 'arterialized' blood.

On the first occasion, volunteers were given $100 \mathrm{~g}$ liquid glucose by mouth as $171 \mathrm{ml}$ of the starch hydrolysate (HyCal; Beechams, Brentford) diluted to $342 \mathrm{ml}$ with water. Venous and 'arterialized' blood samples were collected at frequent intervals before, and for $3 \mathrm{~h}$ following ingestion of glucose. Additional samples of 'arterialized' blood were taken at 5min intervals during the first hour of the experiment in order to provide the information on glucose concentrations which would be matched during the second part of the experiment. Plasma glucose concentrations were measured in all 'arterialized' and venous blood samples; plasma immunoreactive insulin, GIP and C-peptide were assayed in venous blood samples only.

On the second occasion, 1 week later, in addition, a third intravenous cannula was inserted into the opposte arm and a $250 \mathrm{~g}$ glucose/1 solution (Evans Medical, Liverpool) inflused through it. The rate of infusion was constantly adjusted to ensure that the concentration of glucose in the 'arterialized' blood mimicked that achieved during the oral glucose test performed earlier in that individual.

The oral and IV glucose loads were repeated as on the first two occasions after subjects had been consuming the HFD for $28 \mathrm{~d}$. Informed consent was given by all volunteers and the studies were approved by the ethical committees of St Lukes Hospital, Guildford, and Surrey University. 
Table 1. Fat, carbohydrate and energy intake in five healthy volunteers before and during supplementation of their normal diet with $146 \mathrm{ml}$ double cream $\dagger$

(Mean values with their standard errors)

\begin{tabular}{|c|c|c|c|c|c|c|}
\hline & \multicolumn{2}{|c|}{$\begin{array}{l}\text { Total fat } \\
\text { intake } \\
(\mathrm{g} / \mathrm{d})\end{array}$} & \multicolumn{2}{|c|}{$\begin{array}{c}\text { Total carbohydrate } \\
\text { intake } \\
(\mathrm{g} / \mathrm{d})\end{array}$} & \multicolumn{2}{|c|}{$\begin{array}{l}\text { Total energy } \\
\text { intake } \\
(\mathbf{k J} / \mathrm{d})\end{array}$} \\
\hline & Mean & SE & Mean & SE & Mean & $\mathrm{SE}$ \\
\hline $\begin{array}{l}\text { Normal diet } \\
\text { (control) }\end{array}$ & 103 & 9 & 279 & 45 & 9855 & 937 \\
\hline High-fat diet & $170^{* * *}$ & 8 & 259 & 45 & $12347^{* *}$ & 770 \\
\hline
\end{tabular}

Mean values were significantly different from those for the normal diet: ${ }^{* *} P<0.0025,{ }^{* * *} P<0.001$.

$\dagger$ For details of dietary regimen, see p. 374.

\section{Chemical analyses}

'Arterialized' plasma glucose concentrations were measured initially at the bedside, using a Reflocheck Meter (Boehringer, Mannheim, Germany) and these values were used to adjust the rate of glucose infusion. All samples were later re-analysed using an automated glucose oxidase ( $E C$ 1 1 1.3.4) technique and it is these values which are presented. Plasma immunoreactive insulin, C-peptide (Hampton \& Marks, 1979) and GIP (Morgan et al. 1978) concentrations were measured by double-antibody techniques using antisera supplied by Guildhay Antisera (University of Surrey, Guildford). The sensitivity of the insulin assay was $2.5 \mathrm{mU} / 1$, the C-peptide assay $100 \mathrm{ng} / 1$ and the GIP assay $110 \mathrm{ng} / \mathrm{l}$. Interassay coefficients of variation were $7.4,9.0$ and $11.0 \%$ respectively.

\section{Statistical analyses}

Results were compared using Student's $t$ test for paired values (two-tailed). Areas under the curve (AUC) were calculated using the trapezoidal rule. $P$ values of $<0.05$ were accepted as statistically significant.

\section{RESULTS}

Subjects' mean carbohydrate, fat and energy intakes before and whilst consuming the HFD are shown in Table 1. Subjects increased their fat intake by 68 (SE 9.6)\% and their energy intake by 27 (SE 5.4) \% whilst consuming the HFD. They showed a small but significant weight gain after the test period (weight at start of test period 70.6 (SE 1.7) $\mathrm{kg}$; immediately after test period 72.1 (SE 1.6$) \mathrm{kg} ; P<0.025$ ). No significant differences in basal circulating levels of insulin, C-peptide, glucose or GIP were observed after the period of fat supplementation.

Mean plasma GIP concentrations following oral glucose were $50 \%$ higher after subjects had consumed the HFD, compared with the control period (Fig. 1). The incremental AUC for plasma GIP 0-180 min was significantly greater after the HFD compared with the control period (AUC 1660 (SE 592) v. 2642 (SE 750) ng/l.h for control and HFD periods respectively; $P<0.05)$.

The amount of intravenous glucose required to match arterialized plasma glucose levels after oral glucose was similar for the subjects whilst on the normal diet and the HFD (37.2 (SE 2.2) g glucose in control period v. 30.0 (SE 6.2) g glucose after the HFD; not significant). 


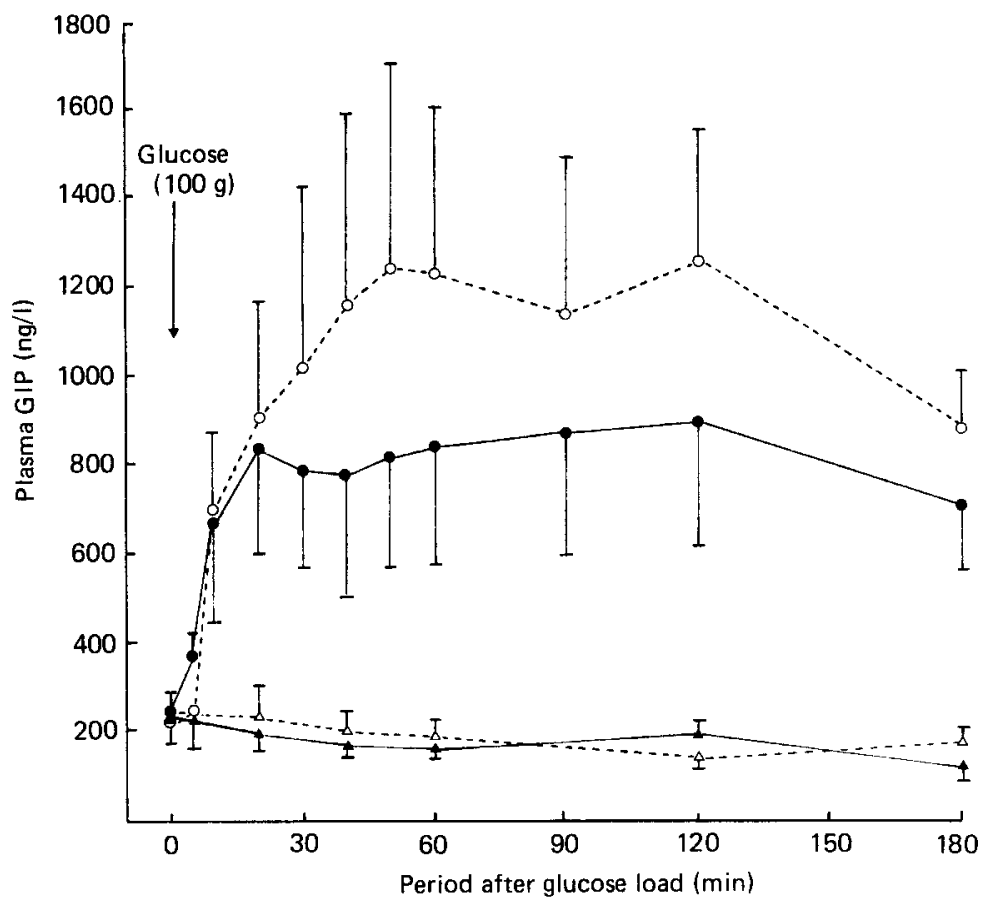

Fig. 1. Plasma gastric inhibitory polypeptide (GIP) concentrations in five healthy subjects following $100 \mathrm{~g}$ oral glucose or intravenous (IV) glucose before, and after $28 \mathrm{~d}$ on a high-fat diet. ( $)$, Oral glucose, control; (O), oral glucose, high-fat diet; (A), IV glucose, control; $(\triangle)$, IV glucose, high-fat diet. Values are means with their standard errors represented by vertical bars. For details of dietary regimens and treatments, see p. 374.

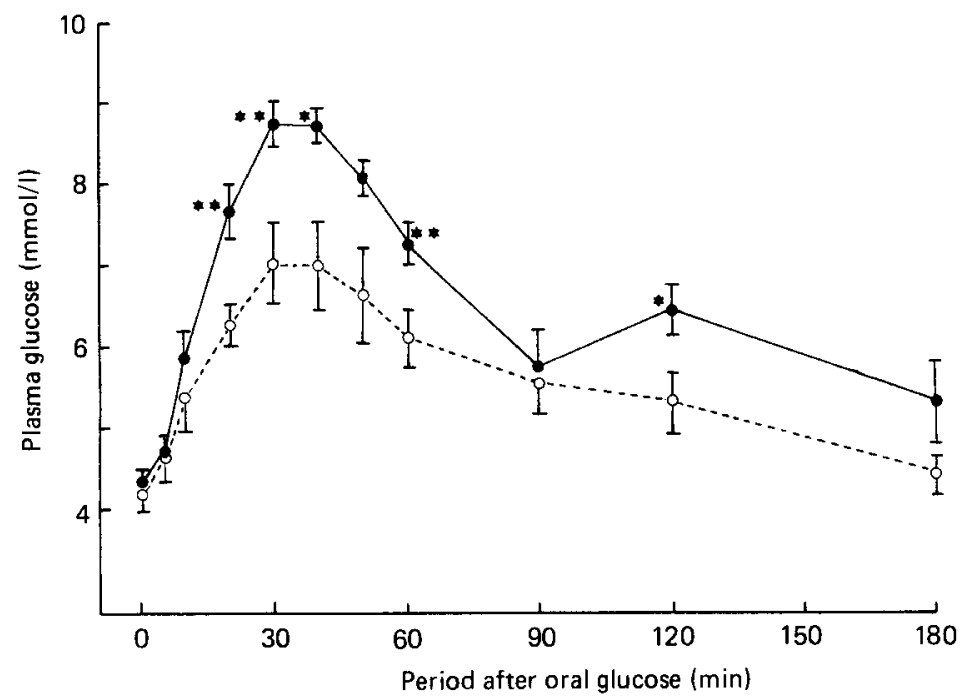

Fig. 2. Arterialized plasma glucose concentrations in five healthy subjects following $100 \mathrm{~g}$ oral glucose before $(O)$ and after $(O) 28 \mathrm{~d}$ on a high-fat diet. Values are means with their standard errors represented by vertical bars. Mean values were significantly different from those after a high-fat diet: ${ }^{*} P<0 \cdot 05$, ** $P<0.025$. 


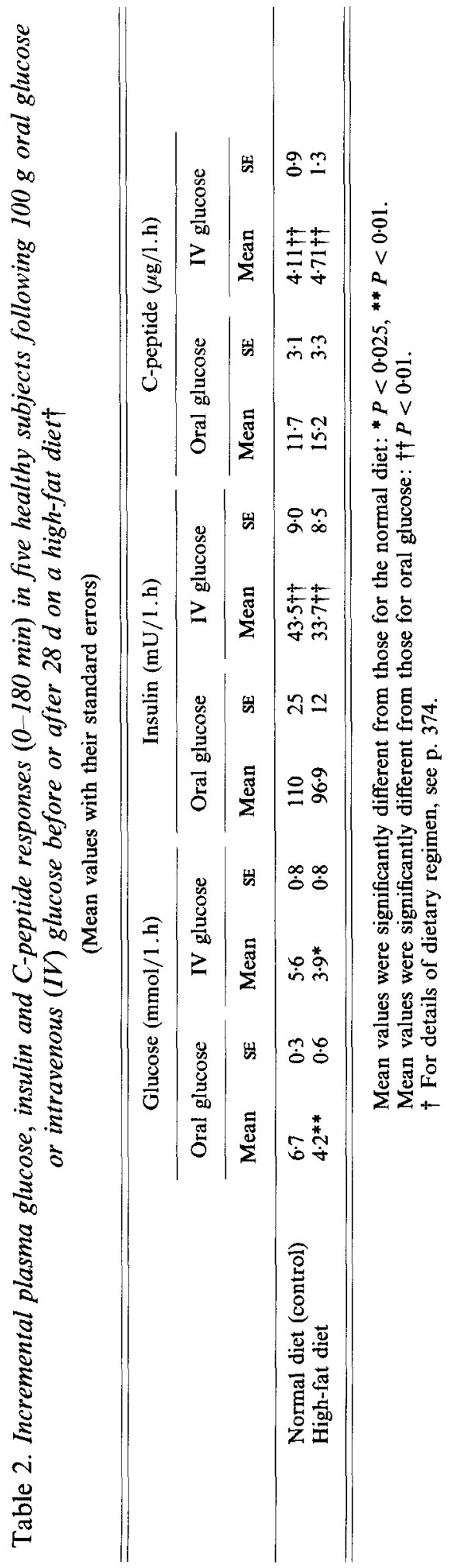


However, with the HFD arterialized plasma glucose levels were significantly lower after oral glucose and this was reflected in the matched IV glucose curves (Fig. 2, Table 2). A similar pattern was observed in the venous plasma glucose levels. Incremental arteriovenous glucose differences after oral glucose were not affected by the HFD.

Insulin secretion in response to glucose was unchanged by the HFD despite an improvement in glucose tolerance and higher stimulated GIP levels. Both incremental insulin and C-peptide responses were significantly higher after oral than after IV glucose $(P<0.01)$ but neither were affected by the HFD (Table 2$)$. Moreover, the HFD caused no change in the 'incretin' effect (that portion of insulin or C-peptide secretion expressed as the difference between insulin secretion after oral, compared with IV glucose) for either insulin or C-peptide.

\section{DISCUSSION}

The present study demonstrates that a 4-week period of dietary fat supplementation in normal subjects results in a modest but significant increase in plasma GIP levels following oral glucose. This finding is in broad agreement with our previous work carried out in rats (Hampton et al. 1983) where a short-term HFD increased circulating GIP levels. A similar increase in post-prandial GIP levels in man has been reported after consuming a diet high in sucrose (O'Dorisio \& Cataland, 1981). Thus, dietary changes appear to be effective in modifying plasma GIP levels and, by inference, GIP secretion, following nutrient stimulation.

A further unexpected finding was the lower circulating glucose levels observed after oral glucose was given to subjects when consuming the fat-supplemented diet. No specific measurements of peripheral glucose uptake were made, but arterio-venous glucose differences were unchanged by the HFD and therefore the improved glucose tolerance was presumably a reflection of differences in hepatic glucose uptake or production rates, or rate of glucose uptake from the gut rather than differences in peripheral glucose utilization. The amounts of IV glucose required to match arterialized plasma glucose levels achieved after oral glucose were similar whether the subjects were fed on a normal diet or the HFD. This was true even though the levels were matched to the lower post-oral glucose levels achieved during consumption of the HFD. The mean amount of IV glucose required to achieve parity with the HFD was, however, $20 \%$ less than that when the subjects were following their normal diet, and failure of the differences to reach statistical significance may merely reflect the large inter-individual variations and small number of subjects involved. Whilst these findings do not exclude the possibility that an HFD induces changes in intravenous glucose tolerance, experiments using an equal amount of glucose administered intravenously on the two dietary regimens would have to be performed to confirm this. Previous reports in the literature have demonstrated a tendency for glucose tolerance to be impaired by high-fat or high-energy diets. Both short-term (Hampton et al. 1983) and long-term (Zaragoza \& Felber, 1972; Collier et al. 1985) feeding of rats on diets high in fat cause impairment of glucose tolerance which is probably related to reduction in hepatic glycogen synthesis due to a decrease in glycogen synthetase activity. In man, there is little information available on the metabolic effects of an increase in dietary fat per se. The effect of short-term overnutrition, with a $70 \%$ increase in fat consumption, on oral glucose tolerance was recently reported with equivocal results, although basal circulating insulin levels were increased (Mott et al. 1986).

Among the possible explanations for the diet-induced improvement in oral glucose tolerance without corresponding hyperinsulinaemia observed in the present study are the following. 
(1) Delayed transit time of the meal through the stomach and small intestine. Ileal lipid perfusion delays gastric emptying and transit of a glucose load with concomitant reduction in postprandial blood glucose levels (McL. Welch et al. 1987). However, it is unlikely that the differences, if any, in the fat content of the lower gut after an overnight fast when subjects were consuming an HFD would be large enough to mediate this effect.

(2) A decrease in the rate of glucose uptake from the small intestine induced by the HFD. Absorption of glucose from perfused jejunum loops of rats fed on an HFD for 3 weeks is slower than that from rats fed on a 'normal' control diet, although the effect is small (Collier et al. 1985).

(3) Decreased glycogenolysis. The increase in dietary fat consumption caused an increase in glucose-induced GIP secretion. GIP has recently been shown to augment insulin-dependent inhibition of hepatic glycogenolysis both in rodents (Hartmann et al. 1986) and man (Elahi et al. 1986). Consequently the higher circulating GIP levels produced by glucose with an HFD may decrease glycogenolysis, which has been postulated to account for at least some of the glucose released into the circulation after ingestion of a glucose load. Although endogenous hepatic glucose production is small after glucose ingestion in man (Jackson et al. 1986) it is very sensitive to changes in insulin concentration (Rizza et al. 1981) and may thus be sensitive to the synergistic action of GIP and insulin.

The fat content of Western diets has recently become a popular focus of attention and evidence has accumulated to implicate the dietary fat content in the aetiology of coronary heart disease. The present study has shown that it is possible to affect GIP secretion in man as in rodents (Flatt et al. 1985) by means of dietary manipulation. Although no effects were seen on either insulin secretion or the entero-insular axis, it is possible that changes in gastrointestinal hormone secretion form the first link in a chain of events that are initiated by changes in diet, and which could eventually lead to systemic changes in metabolism in longer-term studies.

\section{REFERENCES}

Brown, J. C., Dryburgh, J. R., Ross, S. A. \& Dupre, J. (1975). Recent Progress in Hormone Research 31, 487532.

Collier, G. R., Chisholm, K., Sykes, S., Dryden, P. A. \& O'Dea, K. (1985). Journal of Nutrition 115, 1471 1476.

Ebert, R., Willms, B., Brown, J. C. \& Creutzfeldt, W. (1976). European Joumal of Clinical Investigation 6, 327.

Elahi, D., Meneilly, G. S., Hinaker, K. L., Rowe, J. W. \& Andersen, D. K. (1986). Proceedings of the 6th International Conference on Gastrointestinal Hormones, Vancouver, BC, p. 18.

Elrick, H., Stimmler, L., Hlad, C. J. \& Arai, Y. (1964). Journal of Clinical Endocrinology and Metabolism 24, 1078-1082.

Flatt, P. R., Bailey, C. J., Kwasowski, P., Swanston-Flatt, S. K. \& Marks, V. (1985). Nutrition Reports International 32, 847-854.

Gibby, O. M. \& Hales, C. N. (1983). British Medical Journal 286, 921-923.

Hampton, S. M., Kwasowski, P., Tan, K., Morgan, L. M. \& Marks, V. (1983). Diabetologia 24, $278-281$.

Hampton, S. M. \& Marks, V. (1979). Diabetologia 17, 24.

Hampton, S. M., Morgan, L. M., Tredger, J. A., Cramb, R. \& Marks, V. (1986). Diabetes 35, 612-616.

Hartmann, H., Ebert, R. \& Creutzfeldt, W. (1986). Diabetologia 29, 112-114.

Jackson, R. A., Roshania, R. D., Hawa, M. I., Sim, B. M. \& Disilvio, L. (1986). Journal of Clinical Endocrinology and Metabolism 63, 541-549.

McIntyre, N., Holdsworth, C. D. \& Turner, D. S. (1964). Lancet ii, 20-21

McL. Welch, I., Bruce,C, Hill, S. E. \& Read, N. W. (1987). Clinical Science 72, 209-216.

Morgan, L. M., Morris, B. A. \& Marks, V. (1978). Annals of Clinical Biochemistry 15, 172-177.

Mott, D. M., Lillioja,S. \& Bogardus, C. (1986). Metabolism 35, 160-165.

O'Dorisio, T. M. \& Cataland, S. (1981). In Gut Hormones, pp. 269-272 [S. R. Bloom and J. M. Polak, editors]. Edinburgh: Churchill Livingstone. 
Paul, A. A. \& Southgate, D. A. T. (1978). Mc Cance and Widdowsons' The Composition of Foods, 4th ed. London: H.M. Stationery Office.

Rizza, R., Mandarino, L. \& Gerich, J. (1981). American Journal of Physiology 240, E630.

Stout, R. (1982). International Journal of Obesity 6, 111-115.

Willms, B., Ebert, R. \& Creutzfeldt, W. (1978). Diabetologia 14, 379-387.

Zaragoza, N. \& Felber, J. P. (1972). Hormones and Metabolic Research 4, 25-30. 